
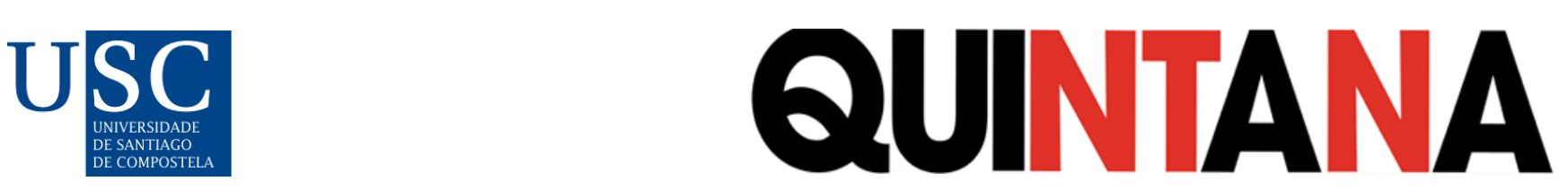

Quintana: revista do Departamento de Historia da Arte, (20), 2021. ISSN-e: 2340-0005

https://doi.org/10.15304/quintana.20.8051

Tema

\title{
LA DIMENSIÓN CULTURAL Y TERRITORIAL DE LOS CAMINOS HISTÓRICOS EN GALICIA Y LOS INSTRUMENTOS PARA LA PROTECCIÓN
}

\section{CULTURAL AND TERRITORIAL DIMENSION OF THE HISTORICAL WAYS IN GALICIA AND ITS INSTRUMENTS FOR PROTECTION}

\author{
Carlos Nárdiz Ortiz ${ }^{1,}$ (iD \\ ${ }^{1}$ Universidade da Coruña, España \\ a carlos.nardiz@udc.es
}

\section{Resumen}

El entendimiento de las transformaciones de la red viaria, y del territorio y el paisaje que soportan en cada momento los caminos históricos, es fundamental para acercarse a su dimensión cultural y territorial, especialmente en Galicia, como en el caso de los Caminos de Santiago, cuya experiencia de delimitación y protección es relativamente reciente y todavía no está finalizada. Esta experiencia es necesario extenderla a otros caminos históricos, sean vías romanas, otros caminos de origen medieval o caminos reales, cuyos instrumentos de protección tienen que relacionarse con la escala territorial de los caminos.

Palabras clave: caminos de Santiago; caminos históricos; territorio; paisaje; instrumentos de protección.

\section{Abstract}

Understanding the transformations of the road network, and of the territory and landscape that the historical roads support at all times, is essential to approach their cultural and territorial dimension, especially in Galicia, as in the case of the Caminos de Santiago, whose delimitation and protection experience is relatively recent and not yet completed. This experience must be extended to other historical roads, be they Roman roads, other roads of medieval origin or royal roads, whose instruments of protection have to be related to the territorial scale of the roads.

Keywords: roads to Santiago; historic roads; territory; landscape; protection instruments.

\section{INTRODUCCIÓN}

Decía Ramón Piñeiro, en relación al paisaje de Galicia que "A nosa paisaxe e natureza humanizada, natureza feita cultura", y a lo mismo se refería Otero Pedrayo, cuando decía que "A paisaxe componse de procesos e formas", de tal manera que como señala Iago Seara la forma del paisaje y el territorio 
que heredamos, fueron conquistados a lo largo de generaciones y generaciones, por lo que la convierten en un bien heredado y transferido. De ahí su dimensión cultural, como soporte de una cultura material e inmaterial (Otero Pedrayo 1955).

Es el papel de los caminos, en la construcción histórica del territorio, más allá de su papel funcional, lo que les convierte en un recurso cultural, y al igual que el territorio, su valoración se relaciona con la capacidad que tenemos de identificar su continuidad histórica, en la que reconocemos la huella de sus trazados, adaptados a la geografía , a las formas de ocupación del territorio, y a las lógicas del transporte en cada momento, hasta el punto de convertirse en elementos estructurantes, cuya desaparición o abandono, determina la desaparición de la estructura territorial que soportan, con su carga paisajística y patrimonial.

La mirada funcional, asociada a los recorridos de pasado entre elementos distantes, sean iglesias, hospitales, puentes o núcleos de población, como ocurre con las delimitaciones que se han realizado de los Caminos de Santiago, confundidos a veces con carreteras o vías locales, olvida a menudo la dimensión cultural de su papel en la construcción del territorio, apoyada también en la falta de entendimiento de las transformaciones de la red viaria.

Ello da lugar a lo que Arturo Soria y Puig llamaba una "visión descaminada de los Caminos de Santiago", en la que se suele traer a colación la unidad europea, el románico, excepto el camino como tal, por la incapacidad de muchos ciudadanos de concebir que quepa viajar a pie o a caballo (Soria Puig 1992).

Decía Xoaquín Lorenzo, que "todo país de montaña e Galicia é o de abondo, adoita ter moitos camiños", en donde la toponimia es rica al designarlos como" curripa, atallo, surreira, eira, sendeiro, carreira, calzada, congostra, camino de herradura, camino de carro, camino francés, camiño vello, camiño de mouros, camino real". Respecto al camino de carro decía, que era más ancho que el de herradura, y que el camino francés, era el recorrido por los peregrinos, que el "camiño vello o de mouros" era el que los campesinos identificaban con las vías romanas, y que los caminos reales, eran los conservados por el Estado, que luego fueron sustituidos por las carreteras (Lorenzo 1982).

El lenguaje de los caminos está cargado de todo tipo de signos, como parte de un sistema territorial, diferenciándose entre aquellos que viven en su cercanía, en donde la variable funcional está presente, de aquellos que los miran con un valor territorial y patrimonial.

No todos estos caminos tienen, en este sentido, la capacidad de estructurar el territorio, y solo los de larga distancia, los que superan la escala de la parroquia y el municipio, y se adentran primero a las villas y luego a las ciudades, poseen esa singularidad. A ellos pertenecen los restos de las calzadas romanas que unían ciudades y explotaciones mineras con el mar, los caminos medievales que unían villas y ciudades de reciente formación, los caminos reales, que transformaron los anteriores caminos de herradura en caminos de ruedas, antes de la construcción de las primeras carreteras modernas a partir de la segunda mitad del siglo XVIII (Nárdiz Ortiz 1992).

El entendimiento de las transformaciones de la red viaria, y del territorio y el paisaje que soportan en cada momento, es fundamental para su identificación y valoración, en donde la traza de los caminos históricos, no está bajo las carreteras modernas, excepto en algún tramo cerca del cruce de un río y de un paso de montaña, a diferencia de lo que ocurre con la señalización que se realizó por ejemplo desde los años 90 de los Caminos de Santiago.

Si la señalización es inadecuada, por desconocimiento o motivos económicos y turísticos, el territorio y el paisaje asociado a los mismos es también inadecuado, en donde el primer paso sería la valoración de la dimensión cultural y territorial de los caminos históricos, especialmente en Galicia, por su papel en la construcción del territorio. 


\section{EL PATRIMONIO DE LOS CAMINOS HISTÓRICOS}

Por caminos históricos entendemos todos aquellos caminos que sirvieron a unas relaciones entre los núcleos de población previas al trazado de las carreteras a partir de la segunda mitad del siglo XVIII.

Incluimos los caminos megalíticos y castreños, identificados a partir de los restos arqueológicos, las vías romanas, cuya continuidad es identificable entre pasos definidos, también a partir de los restos arqueológicos de su explanada, o de las obras de fábrica como los muros y los puentes, los caminos de origen medieval, primero de herradura, y luego transformados con modificaciones parciales de su traza en caminos de ruedas, los propios caminos de ruedas, que se han conservado como memoria de las relaciones entre los núcleos de población y los territorios agrícolas o de monte que dependían de ellos, los caminos reales (fig. 1), construidos también como caminos de ruedas, para servir a territorios distantes, y si nos moviésemos también en el resto de la península, los caminos de la Mesta, o vías pecuarias, recorridos estacionalmente para el pastoreo, entre los agostaderos y los invernaderos.

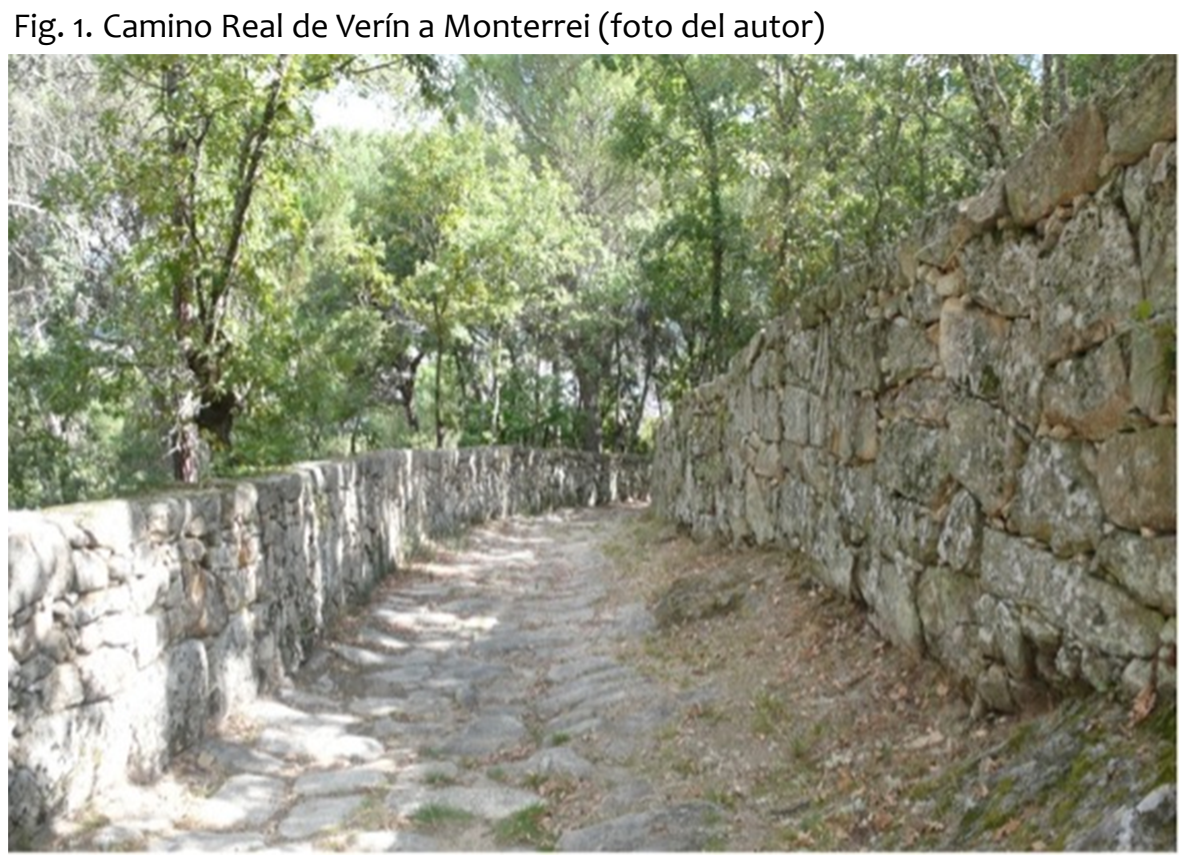

Lo que entendemos hoy por caminos de Santiago, cuando están bien señalizados, no son más que caminos de rueda, que nos han llegado a través de sus transformaciones posteriores(en su origen caminos de herradura), por transformaciones de su ancho, nuevos pavimentos, construcción en sus borde de edificaciones hasta conformar nuevos núcleos de población, con la presencia de cierres de piedra laterales(cuando no han desaparecido),y con trazados adaptados a la geografía, en función del destino final, Santiago de Compostela, para peregrinos y comerciantes.

El patrimonio asociado a estos caminos no es solamente la propia traza, con sus transformaciones posteriores si no también los núcleos de población que han crecido en sus bordes, desde pequeñas aldeas a ciudades, junto a los hospitales, iglesias, ermitas, fuentes, muros laterales y estructuras rurales y urbanas ordenas según la ley del camino. Los cierres, fuentes y árboles que han crecido en sus bordes, los pasos de barca, vados y puentes construidos de forma permanente 
en el cruce de los ríos, el paisaje natural, rural o urbano, con los que nos relaciona su recorrido, forman parte también del patrimonio viario.

El patrimonio asociado a estos caminos históricos son también los itinerarios, guías, mapas, músicas, relatos de viajeros que apoyan la existencia de un determinado camino principal. Cuando la documentación se une a la traza de un camino principal, el valor histórico del camino se une a los núcleos por donde pasa, al puente que le permite cruzar el río, a la franja territorial atravesada. Es el caso de la vía romana de la Plata, del camino francés a Santiago, de la ruta de la seda, de los caminos que siguió el Cid, el Arcipreste de Hita, Cosme de Medicis, Carlos I desde sus desembarcos, el Padre Sarmiento, o los caminos de la poesía de Rosalía de Castro, como el de Bastabales, o el de Antonio Machado, como el de San Saturio, que bordea el Duero en Soria.

Si incluimos también como caminos históricos, las primeras carreteras modernas de la segunda mitad del XVIII, denominadas Caminos Reales y Transversales, tendríamos que referirnos también a la documentación histórica, menos conocida por los historiadores, como es la relativa a los propios proyectos, a las instrucciones y documentos para su construcción y estado de ejecución, que podemos ver por ejemplo en el Archivo de Simancas en Valladolid o en el Militar de campamento en Madrid (Nárdiz Ortiz 1997, 66-75).

Estos proyectos, como ocurrirá también con los de las carreteras del XIX, que podemos encontrar en algunas jefaturas de carreteras y de forma concentrada en el Archivo de la Administración de Alcalá, recogen la traza de los caminos históricos, a los que sustituyeron las carreteras, lo que permite su estudio y la identificación de sus recorridos, en una cartografía que solo existe en relación a los proyectos de las carreteras y en general de las obras públicas(puertos, ferrocarriles, canales, abastecimientos de agua), antes de disponer la propia cartografía de los núcleos de población.

La valoración cultural de este patrimonio de los caminos históricos, aparece condicionada por los problemas que implica su identificación, en el caso de los caminos más antiguos(de origen prerromano, romano y medieval), por la pérdida de su traza y de su continuidad debido a la construcción de carreteras moderna que se han superpuesto en tramos singulares, por la extensión del crecimiento urbano en torno a las ciudades y villas, con la ocupación de los particulares, en el caso que los caminos no hayan sido bien señalizados o delimitados, por la construcción de determinados servicios de abastecimiento, saneamiento, gas, telefonía, o por los procesos de concentración parcelaria, realizados parroquia a parroquia, por ejemplo en Galicia, que han transformado la estructura territorial anterior soportada por los caminos históricos, con sus consecuencias funcionales, patrimoniales y paisajísticas.

Con los caminos históricos ha ocurrido, que, perdido su valor funcional, la Administración no ha tenido interés en conservarlos, y tampoco los particulares cuando no daban acceso a sus parcelas, y solamente han sido recuperados para un uso turístico, como en el caso de los Caminos de Santiago, o las rutas señalizadas para el recorrido de la Naturaleza, que les ha permitido una cierta consideración de su protección y conservación.

\section{LA EXPERIENCIA DEL CAMINO FRANCÉS A SANTIAGO}

La historia se ha ocupado de los Caminos de Santiago, divulgando los primeros itinerarios de los peregrinos más conocidos: Códice Calixtino (S. XII), Nonpart de Caumont (1417), Arnold von 
Harft (1493), Doménico Lafli (1664) que hacen referencia al paso por los principales núcleos de población del Camino Francés.

Esta labor itinerante, ha encontrado en las últimas décadas el soporte de nuevas publicaciones, en las que son precedentes los libros de Vázquez de Parga, Lacarra, Uría (1948), Goicochea Arrondo (1971), que por primera vez recogía una cartografía del Camino Francés a escala, aunque fuese 1/200.000, o Elías Valiña (1985), esta última, haciendo referencia al paso por los núcleos menores en planos o croquis sin escala. Todos ellos se refieren fundamentalmente al Camino Francés (fig. 2). El resto de los libros publicados sobre los otros caminos en forma de guías datan de los años 90 y mayoritariamente de este siglo, en donde se mantienen las carencias de planos de escala adecuada que soporten el recorrido, y los que los recogen son en forma de guías turísticas para orientar al caminante o ciclista en relación a los núcleos por donde pasa y a la cercanía de las carreteras (Vázquez, Lacarra y Uría 1948; Goicoechea Arrondo 1971; Valiña Sampedro 1985).

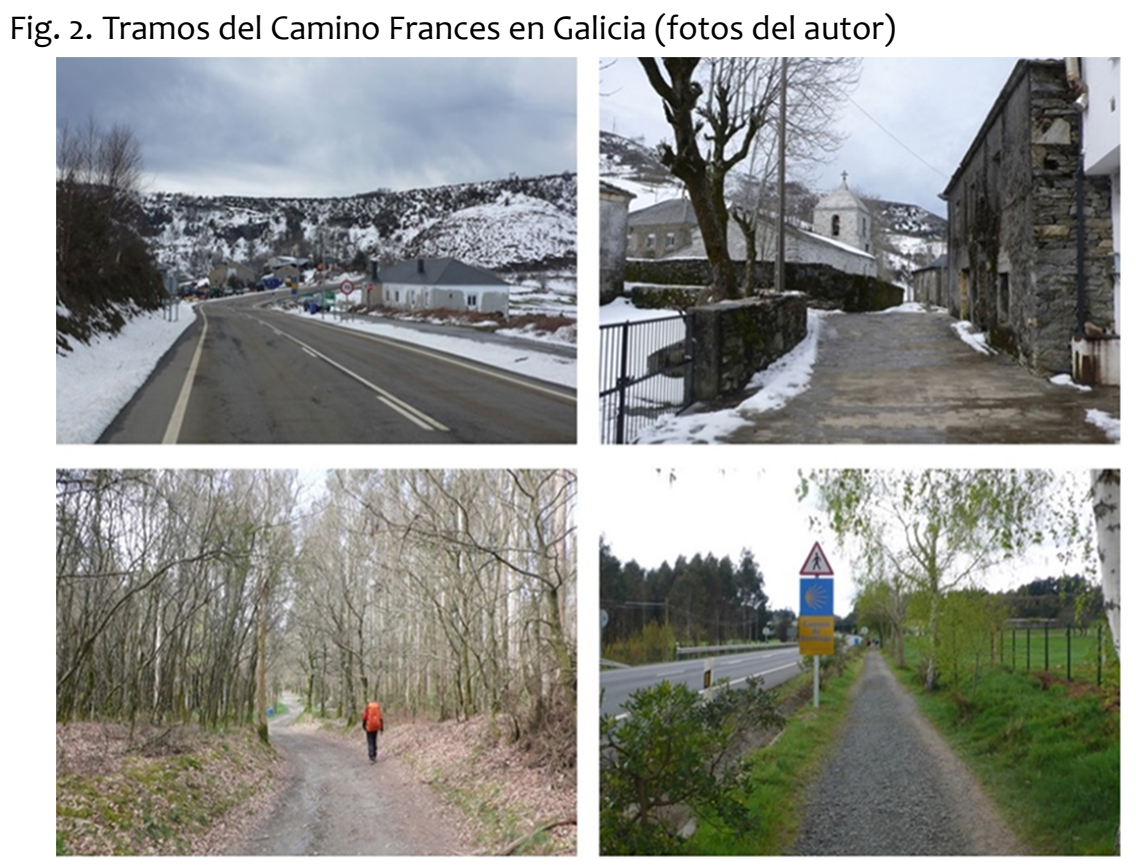

Únicamente habría que destacar entre los precedentes el estudio que realizó en 1985 la entonces Consellería de Ordenación del Territorio e Obras Públicas de la Xunta de Galicia, con el nombre "Rehabilitación del Camino de Santiago" que aporta una cartografía a E1/20.000 del Camino Francés en Galicia. Igualmente, el libro que publicó Arturo Soria y Puig sobre "El Camino de Santiago" $(1991,1992)$ recogía las señales, puentes, fuentes, hospitales y el paso por los núcleos del camino (Rehabilitación 1985; Soria Puig 1992).

Ya en 1962, el Estado había declarado el Camino de Santiago, conjunto histórico-artístico, comprendiendo en esta declaración los lugares, edificios y parajes conocidos en aquel momento, más lo que en el futuro se delimitasen. La Ley 16/1985 de Patrimonio Histórico Español, establecía, que aquellos bienes que fueron declarados como conjuntos históricos artísticos, pasasen a tener la consideración de Bienes de Interés Cultural (BIC) sometido al reglamento jurídico que establece la ley. La declaración de BIC, obligaba a delimitar el ámbito afectado por la declaración.

El apoyo a esta valoración vino también de la declaración en 1984 del Camino de Santiago por parte de la Comunidad Europea como Itinerario Cultural Europeo, que fue refrendada en 1993, con 
la declaración el 17 de mayo como Patrimonio Cultural Europeo y por la UNESCO del 11 diciembre de 1993, como Patrimonio Universal de la Humanidad apoyándose

- En que era una ruta medieval de peregrinos bien identificada.

- En la pervivencia del trazado físico (en un 80 por 100 conservado según ICOMOS, 1992), y de los bienes asociados a su existencia.

- Por constituir una imagen física patrimonial y documentada de una etapa histórica en la que se consolidaron las raíces de Europa.

- Por ser un camino vivo en la actualidad.

El año 1993, coincidió con un año Xacobeo, y con la apuesta por parte de la Xunta de Galicia, a través de la Sociedad de Xestión do Xacobeo para promover el Camino y realizar distintas intervenciones en el mismo que sirvieron para mejorar su traza, aunque en la mayoría de los tramos no coincidiese con la traza histórica.

Antes, la obligación de delimitación al Camino de Santiago como BIC, según la ley de Patrimonio de 1985, llevó a la Xunta de Galicia en 1990 a recoger en las Normas Complementarias e Subsidiarias de A Coruña, Lugo, Ourense y Pontevedra, la obligación de delimitar el ámbito de influencia del Camino de Santiago, con la catalogación e inventario de sus elementos más significativos para el establecimiento de acciones para su puesta en valor. El resultado fue, que en 1991, se declaró el Camino de Santiago, como un conjunto inventariado, estableciendo una franja de $100 \mathrm{~m}$ de protección hasta la aprobación de un Plan Especial de Protección.

Este plan aunque nunca se aprobó, por la oposición de los alcaldes por donde pasaba el Camino, llegó a la fase de avance, realizada por los arquitectos Manuel Gallego y César Portela, que determinó que la Dirección Xeral de Patrimonio Histórico e Documental da Xunta de Galicia, estableciese el 12 de noviembre de 1992, una resolución (DOGA, de 18 de diciembre) en la que incoaba un expediente para la delimitación del Camino de Santiago (Camino Francés), definiendo un trazado y estableciendo un ámbito de protección que llegaba hasta el límite de las parroquias que atraviesa el Camino a lo largo de su recorrido entre Pedrafita del Cebreiro y Santiago de Compostela.

Posteriormente, el 30 de octubre se aprobó la Ley 8/1995 de Patrimonio Cultural de Galicia que tenía como principal novedad añadir a las categorías de BIC (Monumentos, Conjuntos Históricos, Jardines Históricos, Zonas arqueológicas, Sitios de interés etnográfico, Zonas paleontológicas), la de Sitio o Territorio histórico (que ya estaba en la ley de 1985), definido como "un lugar o paraje natural vinculado o acontecimientos o recuerdos del pasado, creaciones culturales o de la naturaleza, y obras del hombre que posean valores históricos o técnicos".

Al año siguiente la Lei 3/1996 de protección do Camiño de Santiago recogía en su artículo 4, la denominación del "Camino de Santiago como un bien de dominio público de carácter cultural incluido en la categoría de territorio histórico, asignando a la delimitación del Camino Francés la delimitación de su territorio histórico realizada en 1992, (es decir el ámbito parroquial atravesado por el Camino) hasta que se aprobase una nueva delimitación. La ley recogía además el procedimiento para la delimitación y deslinde de los Caminos de Santiago, en donde aparte del Camino Francés, recogía los actualmente reconocidos Camino Portugués, Ruta de la Plata, Camino do Norte, Camino de Fisterra, Camino Inglés, y Ruta do Mar de Arousa e Ulla.

La Ley 3/1996 definía además en su artículo 3, el Camino de Santiago como una vía de uso y dominio público, de $3 \mathrm{~m}$. de ancho, y señalaba un ámbito inmediato constituido por un espacio de 
30 m. a partir de los límites exteriores del mismo, que comprendía una zona lateral de protección de $3 \mathrm{~m}$. y una zona de protección del ámbito de $30 \mathrm{~m}$.

Es decir que sobre el Camino o lo Caminos de Santiago, colgaba una doble delimitación

- Por una parte, la integrada por la traza del camino y su territorio histórico.

- Por otra una vía de $3 \mathrm{~m}$. a la que se añadía un espacio de $30 \mathrm{~m}$. con el fin de salvaguardar el BIC.

Para la protección del "territorio histórico" la ley 3/ 1996 trasladaba la protección urbanística recogida en la ley de 1985 para los conjuntos históricos, zonas arqueológicas y lugares de interés etnográfico, a la realización de un Plan Especial de Protección. La Ley de 1996, establecía así mismo un plazo de 2 años para la realización de este Plan que llamaba de "Protección e Promoción do Camiño de Santiago "en el que se dieran audiencia a todos los municipios por los que se pasaba, obligando a que los Planes Especiales que los municipios afectados por el paso del Camino de Santiago pudieran redactar, se adaptasen a las determinaciones el Plan Especial del Camino de Santiago.

Este Plan, que como comentábamos antes nunca se aprobó, implicaba previamente la delimitación de la traza del Camino y de su territorio histórico, de acuerdo con unos pasos que eran recogidos en el artículo 5 de la Ley 3/1996

- Incoación de un expediente de delimitación y deslinde por parte de la Consellería de Cultura, con participación en los municipios atravesados, y en el que intervendrá perceptivamente el Comité Asesor de los Caminos de Santiago.

- Aprobación de la delimitación, por decreto, por parte de la Xunta de Galicia que llevaba asociada la declaración de interés social, la necesidad de ocupación de bienes y derecho a los fines de expropiación forzosa, y la modificación de las servidumbres.

\section{EL PROCESO DE DELIMITACIÓN DE LOS CAMINOS DE SANTIAGO}

A partir de 1993, la Administración, primero a través de la Sociedad Anónima de Xestión do Plan Xacobeo, inició en Galicia un proceso de señalización del resto de los Caminos de Santiago reconocidos documentalmente que atravesaban Galicia, a mayores del Camino Francés. Estos ya habían sido señalizados con la "flecha amarilla" (como la realizada por Elías Valiña) a partir de los años 80, a los que siguieron las Diputaciones Provinciales de Lugo y Ourense, colocando unos primeros mojones en los tramos del Camino Francés. Estos mojones fueron complementados a partir de los años 90, coincidiendo con la preparación del Xacobeo 1993, con nuevas señalizaciones en el Camino Francés por parte de la Administración y a señalizaciones también en el resto de los caminos con grandes errores entonces en la identificación de su traza.

La constatación de estos errores determinó la realización de unos concursos a partir del año 1996, por parte de la Sociedad Anónima de Xestión do Xacobeo, en los que distintos equipos trataron de delimitar los otros caminos, con resultados dispersos, por la falta de entendimiento del trazado de estos caminos de origen medieval, transformados después en caminos de ruedas. Ésta es la señalización que existía hasta que el reconocimiento de las insuficiencias de la misma, hizo que en el año 2005, con el cambio de Administración se planteasen realizar nuevos concursos para la delimitación de la traza y el territorio histórico de los Caminos de Santiago (fig. 3). 
Fig. 3. Territorio Histórico del Camino Frances delimitado

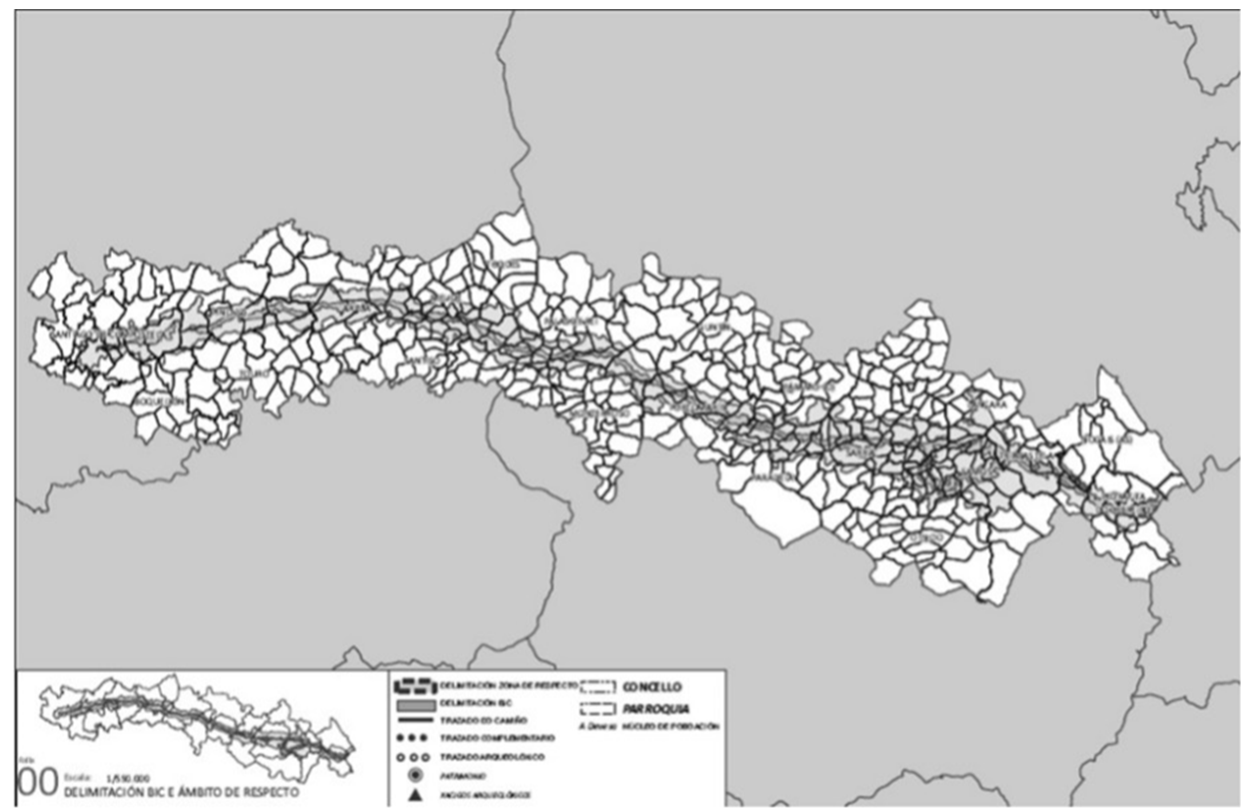

Fuente: Diario Oficial de Galicia, nํ 162, martes, 24 de agosto de 2010

Los estudios se contrataron en el 2007 (Camino Primitivo, Camino do Norte y Camino Francés) y en el 2008 (Camino de Fisterra, Camino Portugués, Camino Inglés y Vía da Plata), esta última dividida en dos tramos: Camino de Ourense a Santiago, Camino tradicional o actual itinerario desde Chaves, que se entregaron en el año 2009.

De todos estos caminos los únicos que tienen aprobada en estos momentos su delimitación son el Camino Francés (2011-2012), el Camino del Norte interior o Camino Primitivo y el Camino de la Costa (2012), el Camino Inglés (2012), y el acceso del resto de los Caminos a Santiago de Compostela. En el expediente de delimitación de estos caminos, como en el caso del Camino Francés, se distingue entre la traza y el ámbito de delimitación del BIC.

Respecto a la traza delimitada se diferencia entre:

- Trazado del camino, es decir, el itinerario principal, al que asociaba a la delimitación que establecía la Ley 3/1996.

- Trazado complementario, en el que se incluyen aquellos tramos del camino que tienen una certeza menor que el principal, y que no tiene suficiente rigor histórico a pesar de estar hoy consolidado.

- Trazado arqueológico, en el que se señala trazados que tienen un carácter histórico, aunque hoy ya no se pueden utilizar.

Se parte para ello de que el trazado del camino, no es unitario, sino que está constituido por una red de caminos o ramales, utilizados según las distintas épocas del año, por lo que se considera que se pueden utilizar varias alternativas al itinerario principal.

Para la identificación del itinerario principal, que asimila al trazado histórico, se apoyan en criterios como

- Optar por el trazado actualmente consolidado cuando existe igual grado de certeza. 
- Dar continuidad al camino, desde el punto de vista funcional, cuando el histórico se ha perdido.

- Considerar irrecuperables aquellos tramos desparecidos bajo una infraestructura o edificio.

- Considerar que tanto el camino histórico identificado, pero irrecuperable, como los otros caminos históricos documentados, deben ser señalizados como territorios históricos o arqueológicos.

Estos criterios fueron criticados entonces por los errores graves que tenía la delimitación propuesta, por no ser más estrictos con el trazado histórico, que podría derivar en la destrucción de tramos que son Patrimonio de la Humanidad. La crítica fundamental era que el itinerario principal había sido seleccionado con criterios de "consolidación" por el uso, y no por su autenticidad histórica. La naturaleza histórica del territorio asociado a los Caminos de Santiago, no cabe entenderla más que como una estructura de un complejo agrario de origen medieval, contemporáneo a la formación del camino. Yo mismo realicé un informe apoyado en cartografía $\mathrm{E}$ $1 / 5.000$, con el parcelario incluido y documentos fotográficos de la traza del Camino Francés, en el que ponía de manifiesto las contradicciones del expediente de delimitación (Bau-Signo 2008; Nárdiz Ortiz 2010).

El criterio utilizado por la Ley 3/1996, que asimila los Caminos de Santiago a aquellas rutas históricas reconocidas documentalmente, está en contradicción con los estudios realizados hasta entonces sobre una cartografía inadecuada, por lo que este reconocimiento documental no es posible más allá del paso por los núcleos identificados por distintos autores, entre los que se ponían de manifiesto contradicciones.

Ante esta indeterminación entre núcleos (pero también en los propios núcleos, más allá de la rúa vella o mayor) solo está la propia traza en un territorio transformado por la construcción de la carretera a partir de la segunda mitad del siglo XVIII (en el Francés del XIX), por los procesos de concentración parcelaria, por la apertura de nuevas calles en la periferia de las villas y ciudades, por la transformación del ancho y el pavimento con las demoliciones de los cierres y edificaciones anteriores que bordeaban el camino. Cuando éste se ha conservado en el paso de los núcleos menores, ésta es la mejor evidencia. La traza histórica ha sido ocupada también en algunos casos por los particulares (cuando se ha perdido el paso por la traza histórica), por la construcción de determinados equipamientos deportivos o por nuevas urbanizaciones.

Defendía en este sentido, que cuando se conserva la traza, es posible identificarla, pero no la del camino medieval (excepto en el paso de los núcleos) que era fundamentalmente un camino de herradura, sino la del camino de ruedas que se superpuso sobre ella ensanchándola, o con pequeñas modificaciones de trazado, y que ya aparecía identificada en los itinerarios de la época como el de Villuga.

La identificación de esta traza sólo es posible a través del recorrido directo por la misma, andando, con una cartografía además preparada para ello, fundamentalmente a E 1/5.000 (y $1 / 1.000$ en el paso por los núcleos) con la estructura parcelaria integrada.

Respecto al Ámbito, se modificaba el anterior ámbito parroquial como territorio histórico al que se asociaba la delimitación del BIC de 1992. Este cambio se apoyaba en análisis visuales y paisajísticos. Parecía, en este sentido necesario, un análisis morfológico, que apoyase la lectura histórica, que no se encuentre en el estudio realizado. 
Del resto de los caminos, los estudios realizados entre el año 2008 y 2009, se fueron revisando posteriormente, por haber considerado la Dirección Xeral de Patrimonio Cultural, que tenían errores importantes en la propia traza señalizada. Finalmente, como antes comentábamos, se aprobaron en el 2012 los expedientes de delimitación del Camino Inglés y de los Caminos del Norte, el Primitivo y el de la Costa.

La presión de los particulares, en donde la Administración está protegiendo tramos mal delimitados (los propios vecinos los saben), dan lugar a cambios de delimitación como en el caso del Camino Inglés entre Santiago (Meixónfrío) y Sionlla, a partir de un estudio que realizamos en febrero de 2009, y que aprobó después la Dirección Xeral de Patrimonio Cultural (Nárdiz Ortiz 2009).

La metodología de este estudio se apoyaba

- En la cartografía histórica existente.

- En la elaboración de una cartografía actual que integrase el viario, el parcelario y sus transformaciones desde los años 50 (a partir del vuelo americano), antes de que actuase la concentración parcelaria.

- En el recorrido de trazados alternativos, al propio camino delimitado.

- En la búsqueda de una traza del camino histórico que se aproxime al original (cuando estos han desaparecido para las carreteras o la concentración parcelaria).

Esta metodología, fue el soporte del estudio que realizamos después para el Camino Francés (fig. 4), y parece la adecuada para las delimitaciones del resto de los caminos, aunque la Administración no considera en estos momentos seguir con las delimitaciones. En la propia página web de la Consellería de Cultura es posible hoy relacionarse con las trazas propuestas para el resto de los caminos.

Fig. 4. Paso por Portomarín del Informe sobre la Traza delimitada del Camino Frances (Carlos Nárdiz 2010)

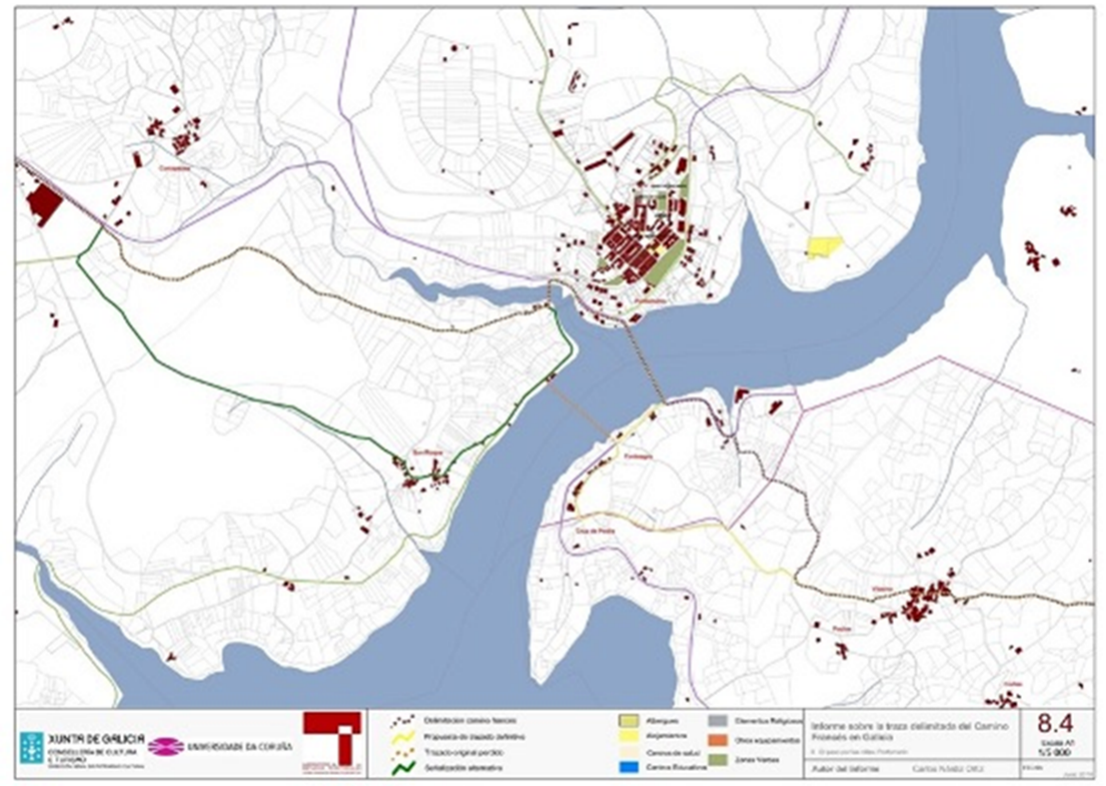




\section{MEDIDAS PARA LA CONSERVACIÓN Y PROTECCIÓN DE LOS CAMINOS DE SANTIAGO}

A los problemas de delimitación de la traza y del ámbito, se unen las medidas para la conservación y protección de los Camino de Santiago, con la franja de protección y el territorio histórico, que van desde la limitación de uso de la traza y zonas laterales, hasta la protección urbanística del territorio histórico y de cada uno de los elementos incluido en el mismo, entre los que se encuentran bienes declarados de interés cultural (BIC).

La Lei 3/1996 de protección dos Camiños de Santiago distinguía entre tramos urbanos y no urbanos, considerando los primeros, los tramos de los caminos que discurran por tramos clasificados por el planeamiento como urbanos.

En los tramos no urbanos, los caminos aparecen delimitado por la franja lateral de protección, que se sitúa a $3 \mathrm{~m}$ de la arista exterior de los caminos. La explanada de los Camino de Santiago se prohíbe que sea utilizada para el tráfico rodado, excepto cuando sirva de acceso a los núcleos o a viviendas. En la zona lateral de protección, cualquier uso debe ser autorizado por la Consellería de Cultura, quedando prohibida cualquier actividad constructiva o la poda de árboles.

Además, como comentábamos antes, se establece una franja de protección del contorno, compuesta por dos franjas de $30 \mathrm{~m}$ de ancho, contando desde el borde de los caminos, en las que los usos quedan sometidos a la autorización de la Consellería de Cultura, previo informe del Comité Asesor del Camino de Santiago. En estas franjas se prohíbe todo tipo de publicidad, la explotación minera, la extracción de gravas o arenas.

La ley anterior de Patrimonio Cultural de Galicia de 1995, establecía la protección urbanística del territorio histórico del camino previamente delimitado, a través de la realización de un Plan Especial de Protección e Promoción dos Camiños de Santiago, cuya obligatoriedad decía la ley, no podrá excusarse en la existencia de otros planeamientos. Las determinaciones a este Plan (nunca realizado como antes comentábamos, aunque sí iniciado) estaban llamadas a condicionar las alternativas de los Planes Generales municipales. Mientras no se redactase la ley previa que las actividades y licencias autorizadas que afectasen a los Caminos de Santiago, y a su zona lateral de protección y contorno, necesitarían la resolución previa por parte de la Consellería de Cultura, y el informe preceptivo del Comité Asesor del Camino de Santiago.

Se entiende, en este sentido, que la protección del Camino o Caminos de Santiago necesitan toda una administración paralela que cuelgue de la Consellería de Cultura de la Comunidad Autónoma, o también la Dirección Xeral de Patrimonio Cultural, y que en Galicia incluye también el Comité Asesor do Camiño de Santiago, y el Consello de Cultura Galega. A mayores está (o estaba) la S. A. de Xestión do Plan Xacobeo (creado en 1991) para la rehabilitación y recuperación del camino.

La figura del Plan Especial para proteger el territorio histórico asociado al Camino de Santiago, ha chocado con la voluntad de la Administración para realizarlo, por su carácter supramunicipal, y por la existencia de una legislación de Ordenación del Territorio, que incluye un instrumento más adecuado, en este caso, como son los Planes Territoriales, como el que se realizó, por ejemplo, para la ordenación y las normas de protección del Canal de Castilla en la Comunidad de Castilla y León (Canal de Castilla 2004).

Las Directrices de Ordenación de Territorio de Galicia, aprobadas en el 2011,siguiendo la Ley de Ordenación del Territorio de 1995,seguían defendiendo la figura del "Plan especial de protección de los Caminos de Santiago", como figura más adecuada para la protección del territorio histórico 
asociado a los caminos delimitados, hasta que el título VI de la nueva ley 5/2016 de Patrimonio Cultural de Galicia, estableció como instrumento de protección el Plan Territorial Integrado de los Caminos de Santiago, para la protección de su territorio histórico, de acuerdo todavía con la anterior ley de Ordenación del Territorio de 1995.

La nueva Ley 1/2021 de Ordenación del Territorio de Galicia, aunque mantiene la figura anterior como instrumento de ordenación de áreas supramunicipales que mantienen características homogéneas, reserva a los Planes Territoriales Especiales la ordenación de áreas patrimoniales, paisajísticas y ecológicas diferenciadas, a las que, aunque no los cita, se adaptarían los territorios históricos en torno a los Caminos de Santiago.

Finalmente entró en vigor el 20 de febrero del 2021 el Decreto 238/2020 por el que se aprueban las Directrices Paisajísticas de Galicia, que están llamadas a condicionar la mayor parte de las intervenciones de transformación del territorio con consecuencias paisajísticas ,y que incluyen referencias al patrimonio cultural material e inmaterial de las Sendas, Caminos de Santiago y viarios históricos y tradicionales, como articuladores del territorio, fomentando el conocimiento de sus trazas y de las leyendas y tradiciones relacionadas con los mismos.

El artículo 4.2.4.8 relativo a los Camiños de Santiago, Sendas e Viarios históricos e tradicionais establece limitaciones a las intervenciones en las zonas visibles desde al menos $500 \mathrm{~m}$ de los bordes de los caminos, que requerirán la correspondiente autorización, con la necesidad de establecer la cuenca visual de estas intervenciones, proponiendo crear una red de itinerarios de interés paisajístico y ambiental, preservando el patrimonio construido en su entorno.

En cualquier caso, es de la identificación adecuada de la traza, de la que cuelgan las franjas laterales, las franjas del contorno ,el propio territorio histórico y las zonas visibles ,el paso más importante para la protección de los caminos y en especial los de Santiago, en donde las malas delimitaciones realizadas en la segunda mitad de los 90, que sirvieron para la colocación de mojones y una cierta consolidación de los Camino de Santiago en los ayuntamientos atravesados, están pesando como una losa para tomar decisiones de cambio de la traza. Si a ello unimos las decisiones también equivocadas (en contra a veces de los conocimientos de los vecinos, que saben perfectamente por donde iba el Camiño Vello) se está produciendo una cierta desafección, con sus consecuencias para la protección sobre los caminos actuales, más allá de su interés turístico.

\section{LA PROTECCIÓN DEL RESTO DE LOS CAMINOS HISTÓRICOS}

La asimilación que se ha producido entre los caminos históricos y los Caminos de Santiago, con el apoyo de la Administración para su señalización o recorrido, por su interés turístico o económico, ha hecho olvidar el resto de los caminos, previos a los de Santiago, como las vías romanas, los coetáneos con los de Santiago, como toda la red de caminos medievales que comunicaban monasterios, villas y ciudades sin pasar por Santiago, o posteriores de los caminos de ruedas y reales a partir del siglo XVIII.

Cuando alguno de estos caminos se han podido beneficiar de "pedigrí" de conducir también a Santiago, como el estudiado por Yolanda Barriocanal en su libro sobre $O$ Camiño Real de Castela a Santiago, con sus variantes de la "Verea da Serra Seca" entre A Gudiña a San Cibrán das Viñas, (aunque el verdadero camino de origen medieval es el que venía desde Zamora y Braganza, hasta Verín y Ourense por Vilardevós, como se traduce en la propia estructura histórica de Verín), la Administración los ha señalizado, y ha contribuido aparentemente (digo solo aparentemente 
porque hay tramos muy mal señalizados) a la conservación del camino, al menos en los tramos de los pasos de los puertos, puentes y núcleos de población. En las zonas de valle, el camino ha visto arrasada su traza por la concentración parcelaria (Barriocanal López 2014).

Una vía romana como la Vía XVIII o Vía Nova, que divide transversalmente a Galicia por el Sudeste desde el paso da Portela de Home, a través de la Limia y el valle de Valdeorras, no tiene ningún tipo de protección a pesar de los estudios que se han realizado de la misma, empezando por el de un ingeniero de caminos Díez Sanjurjo, en el siglo XIX. Ni siquiera en el inventario de patrimonio histórico, que la Dirección Xeral de Patrimonio Cultural, remitió a la Consellería de Medio Ambiente para las Directrices de Ordenación del Territorio aparece ninguna referencia a la Vía XVIII, y no digamos al resto de las vías romanas en Galicia. Sería necesario en Galicia, el realizar una delimitación sobre cartografía adecuada de los tramos de las vías romanas que se conservan (Alvarado, Rivas y Vega 1998).

Igual ocurre con los caminos de ruedas que servía al comercio, como el que comunicaba Pontevedra con Berducido, del que se recibió en el Consello de Cultura Galega un informe de la destrucción que se produjo recientemente de este camino real, en el que se han destruido cierres y la pavimentación del propio camino. Los ejemplos de caminos reales bordeados de cierres de piedra, que atravesaban las calles de los principales núcleos formados en torno a ellos, podrían extenderse a cientos, si pensamos en lo que ocurrió a partir de los años 80, en los que la intervención de los Ayuntamientos, con el apoyo fundamental de las Diputaciones Provinciales ensanchó y pavimentó este patrimonio viario, en donde existan otras alternativas para el acceso a los núcleos.

Es verdad que, en Galicia, a partir del Inventario de Pontes Históricas de Galicia, que realizamos desde 1985, y que dio lugar al libro Pontes Históricas de Galicia (1989) ha habido una mayor sensibilidad para el patrimonio de los puentes históricos, fundamentalmente en piedra, y alguno metálico e incluso de hormigón. No así con las pontellas que servían de continuidad para los pequeños arroyos a los caminos parroquiales o reales, que están todos en la memoria de las gentes del lugar (Alvarado, Durán y Nárdiz 1989).

Entre estos caminos, hay que destacar, como comentábamos los Caminos Reales construidos en la segunda mitad del XVIII, con sus fuentes y árboles laterales, junto con los pavimentos que bordean las edificaciones, que han desaparecido, o que se conservan en ruinas como en Oseiro, por un mal entendimiento de la urbanización. Todavía se puede conservar algo de estos tramos, como en el que atravesaba el Puente Cruzul, o el que subía de Os Nogais al puerto de Pedradita (Nárdiz Ortiz 1992).

¿Qué tipo de protección habría que realizar para estos caminos? Bastaría con identificarlos y cartografiar su traza, difundiéndola a través de cartografía y guías, hasta que pueda ser reconocida, de tal manera que en los mismos se produzcan un proceso parecido al que se ha producido con los Caminos de Santiago. En este proceso los Ayuntamientos, e incluso las Diputaciones Provinciales, apoyándolos, pueden contribuir a la difusión de estas rutas, con un interés turístico, de los que se demuestran beneficios económicos para las poblaciones atravesadas.

¿Sería utópico esperar en el futuro una legislación protectora, que abarcara todos los caminos históricos, incluidos los de Santiago, a medida que nuevos caminos fueran progresivamente documentados, que fuese más allá de la declaración genérica de las recientes Directrices de Paisaje, como elementos fundamentales en la construcción del territorio y el paisaje de Galicia? Este es el reto en el que estamos. 


\section{REFERENCIAS}

Alvarado, Segundo, Manuel Durán, and Carlos Nárdiz. Pontes Históricas de Galicia. Santiago de Compostela: Colegio de Ingenieros de Caminos, Canales y Puertos. Xunta de Galicia, 1989. 3ª edición, 1991.

Alvarado, Segundo, Juan Carlos Rivas, and Tomás Vega. La Vía Romana XVIII (Vía Nova). Revisión de su trazado y mensuración. Ourense: Boletín Auriense. Anexo 9, 1998.

Barriocanal López, Yolanda. O Camiño Real de Castela a Santiago por Ourense, base del camino de peregrinación. Vía da Prata. Ourense: Museo Aquivo de Ourense, 2014.

Bau-Signo. Estudio Histórico del Camino Francés. Santiago de Compostela: Consellería de Cultura e Turismo. Xunta de Galicia, 2008.

El Canal de Castilla: un Plan Regional. Madrid: CEHOPU. Junta de Castilla y León, 2 vols., 2004.

Goicoechea Arrondo, Eusebio. Rutas Jacobeas. Historia, Arte y Caminos. Estella: Los Amigos del Camino de Santiago, 1971.

Lorenzo, Xoaquín. A Terra. Vigo: Ed Galaxia, 1982.

Nárdiz Ortiz, Carlos. El Territorio y los Caminos en Galicia. Planos Históricos de la Red Viaria. Madrid: Colegio de Ingenieros de Caminos, Canales y Puertos. Xunta de Galicia, 1992.

Nárdiz Ortiz, Carlos. “Los Caminos Españoles anteriores a 1900.” Revista OP, no. 41 (1997): 66-75.

Nárdiz Ortiz, Carlos. Estudio del trazado del Camino histórico de Ponte Sigüeiro a Santiago de Compostela. A Coruña: Universidad de A Coruña. Laboratorio de Estudios Territoriales. Dirección Xeral de Patrimonio Cultural. Abril, 2009.

Nárdiz Ortiz, Carlos. Informe sobre la traza delimitada del Camino Francés en Galicia. Santiago de Compostela: Consellería de Cultura e Turismo. Dirección Xeral de Patrimonio Cultural. Xunta de Galicia. Junio, 2010.

Otero Pedrayo, Ramón y otros. Paisaxe e Cultura: ensaios. Vigo: Ed Galaxia. 1955. 2ª Ed. 2009, Vigo; Centro de Estudios Oterianos. Limiar, Iago Seara.

Rehabilitación del Camino de Santiago. Santiago de Compostela: Consellería de Ordenación e Obras Públicas. Xunta de Galicia, 1985.

Soria y Puig, Arturo. El Camino de Santiago, Tomo I y Tomo II. Madrid: MOPT, 1991-1992.

Valiña Sampedro, Elías. El Camino de Santiago. Guía del Peregrino. León: Everest, 1985.

Vázquez de Parga, Luis, José María Lacarra, and Juan Uría. Las peregrinaciones a Santiago de Compostela. Madrid: Consejo Superior de Investigaciones Científicas. 1948. 2ª edición. Pamplona: Red del Gobierno de Navarra, 1992. 